\title{
Postoperative Behandlung einer mediokarpalen Teilarthrodese
}

\author{
Ingmar Vander
}

\author{
Nach einer Handgelenksverletzung kann eine Teilversteifung nötig werden, wenn Beschwer- \\ den durch arthrotische Prozesse zunehmen und bewegungserhaltende Operationsmetho- \\ den nicht mehr möglich sind. Handtherapeut Ingmar Vander stellt die postoperative \\ Behandlung eines Klienten mit mediokarpaler Teilarthrodese bis zum Abschluss der \\ 14. Woche vor und beschreibt, wie es seinem Klienten gelungen ist, durch interdisziplinäre \\ Zusammenarbeit von Therapeuten und Ärzten wieder am Leben teilzunehmen.
}

Betätigung ist aus ergotherapeutischer Betrachtungsweise für den Menschen die wichtigste Kernkompetenz eines selbstverwirklichten Lebens. Durch individuelle, sinnvolle Handlungen in den Bereichen des täglichen Lebens erfährt der Mensch körperliche und mentale Gesundheit. Im Umkehrschluss kann eine Beeinträchtigung der Gesundheit negative Auswirkungen auf unser selbstbestimmtes Handeln nehmen. Wie kann ein Mensch, der sich einer bewegungslimitierenden Handgelenksoperation unterziehen musste, die Teilhabe am Leben wiedererlangen?

\section{Der Klient}

Karl B. ist 59 Jahre alt und seit vielen Jahren als Schuhmacheraltgeselle tätig. In seiner Freizeit betreibt er intensives Fitnesstraining und unternimmt viele Angelausflüge. Seit ca. eineinhalb Jahren hat er zusätzlich das Bogenschießen für sich entdeckt. Neben regelmäßigen Fahrradtouren bewältigt er auch im Alltag alle Wege mit dem Fahrrad. All dies ist seit Anfang Januar 2019 nicht mehr möglich. Er hatte einen folgenschweren Fahrradunfall, der sein bisheriges Leben veränderte.

Während einer Fahrradtour stürzte er und fing sich reflexartig mit beiden Armen ab. In der darauffolgenden handchirurgischen Untersuchung im Krankenhaus zweieinhalb Wochen später konnten Frakturen ausgeschlossen werden. Allerdings zeigte das Röntgenbild eine hochgradige Arthrose zwischen dem Os scaphoideum, Os trapezium und dem Os trapezoideum (STT-Gelenk) und der Verdacht auf eine Läsion des skapholunären Bandes (SL-Band) verhärtete sich. Durch eine Arthroskopie Ende Januar wurde diese Diagnose gesichert. Das SL-
Band war komplett rupturiert und die Anteile in einem zu schlechten, aufgefaserten Zustand, um sie durch eine Naht chirurgisch rekonstruieren zu können. Ein plastisches Verfahren konnte aufgrund der stark arthrotischen Prozesse und deren degenerativen Auswirkungen auf die Knochensubstanz ebenfalls nicht angewandt werden. Da er in seinem Beruf voll belastbar sein muss und ohne Operation von weiterer Verschlechterung auszugehen war, rieten ihm die Chirurgen zu einer mediokarpalen Teilarthrodese.

\section{Warum ist eine Operation notwendig?}

Ein intaktes SL-Band ist für einen reibungslosen Bewegungsablauf der proximalen und distalen Handwurzelreihe grundlegend. Diese können als funktionelle Einheit betrachtet werden [1]. Das bedeutet, dass die Verschiebung der Handwurzelknochen durch eine ausgeführte Bewegung eine mechanische Auswirkung auf die jeweils benachbarten Knochen hat. Das SL-Band gibt die nötige Stabilität bei den komplexen Bewegungen des Handgelenks und verhindert eine Subluxation des Os scaphoideum nach palmar und des Os lunatum nach dorsal. Fehlt die ligamentäre Verbindung, vergrößert sich der Abstand zwischen den Knochen zunehmend und die Kinematik verändert sich. Es kommt zu einer „dorsal intercalated segmental instability“, kurz DISI. Im Röntgenbild zeigt sich dabei eine Vergrößerung des skapholunären Winkels über $60^{\circ}$ und des radiolunären Winkels über $30^{\circ}$. Im Röntgenbild mit Faustschluss, einer sogenannten Stressaufnahme, ist dabei der Abstand zwischen 
Skaphoid und Lunatum größer als $3 \mathrm{~mm}$. Durch diese Fehlstellung kommt es zu einer vermehrten Abnutzung der Gelenkflächen, was die Entstehung einer schmerzhaften Arthrose zur Folge hat. Dies wird als SLAC-Wrist bezeichnet (Scapholunate Advanced Collapse). Klinisch zeigen sich eine Bewegungseinschränkung im mediokarpalen Gelenk und ein deutlicher Kraftverlust.

\section{Die Operation}

Einige Wochen nach dem Unfall klangen die Sturzbeschwerden zunächst $a b$ und der Patient nutzte sein Handgelenk wieder uneingeschränkt. Infolgedessen verschlechterte sich der Zustand seiner Hand allerdings wieder: Die Schmerzen nahmen zu, es entwickelten sich eine ausgeprägte Synovialitis der Strecksehnenfächer und eine aktivierte Arthrose. Letztlich konnte er seinem Beruf und seinen Freizeitaktivitäten nicht weiter nachgehen.

Am 14. Mai 2019 wurde somit die mediokarpale Teilarthrodese mit Denervierung sensibler Nerven durchgeführt. Es erfolgte eine $10 \mathrm{~cm}$ lange Inzision auf dem dorsalen Handgelenk. Der Handchirurg eröffnete das Retinaculum extensorum über dem fünften Strecksehnenfach und führte eine Synovialektomie der Sehnen aus dem zweiten bis fünften Strecksehnenfach durch. Anschließend erfolgten die Koagulation und Resektion der Nn. interosseus posterior et anterior. Nach dem Eröffnen des Handgelenks über dem dorsalen Bandapparat zeigten sich Arthrosen im Mediokarpalgelenk und Radioskaphoidalgelenk. Das Skaphoid wurde vollständig entfernt und diente im weiteren Verlauf der Spongiosaentnahme. Nach Entknorpelung der Gelenkflächen des Kapitatumkopfes, des Os hamatum, des Os triquetrum und des Os lunatum erfolgte das Einbringen von Spongiosa im Sinne einer freien Spongiosaplastik. Insgesamt brachte der Chirurg fünf Kirschner-Drähte ein, um die Reposition des Lunatums aus der DISI-Stellung durchzuführen und die umliegenden Handwurzelknochen zu fixieren. Gemeinsam mit den Handchirurgen haben wir in unserer Praxis folgendes Nachbehandlungsschema für Patienten nach mediokarpaler Teilarthrodese ausgearbeitet ( $\mathbf{T a b} \mathbf{1}$ )

\section{ZUSATZINFO}

Zu den möglichen Komplikationen der OP zählt u.a., dass das Material in den Beugesehnenkanal ragt und die Flexorensehnen schädigen kann. Eine Auswertung des Röntgenbilds kann hier aufklärend sein. Dorsalseitig hingegen ist das Material zumindest teilweise gut palpierbar.
-Tab. 1 Praxisinterne Nachbehandlungsrichtlinien für eine mediokarpale Teilarthrodese [1]

\begin{tabular}{|l|l|}
\hline Woche & Therapeutische Maßnahmen \\
\hline $1-6$ & $\begin{array}{l}\text { i.d. R. Ruhigstellung für sechs Wochen, } \\
\text { aktives Bewegen der Finger, des Ellenbo- } \\
\text { gens und der Schulter durch den Patien- } \\
\text { ten, Hochlagern des Armes (über } \\
\text { Herzhöhe), um Schwellungen zu verhin- } \\
\text { dern bzw. zu verringern, ggf. begleitend } \\
\text { antiphlogistische Therapie }\end{array}$ \\
\hline $\begin{array}{l}\text { Beginn der therapeutischen Narbenbe- } \\
\text { handlung, aktive Bewegungsübungen, } \\
\text { abschwellende Maßnahmen, Weichteil- } \\
\text { techniken, schmerzlindernde Techniken } \\
\text { ggf. unter Einbezug der gesamten obe- } \\
\text { ren Extremität und Brustwirbelsäule }\end{array}$ \\
\hline $\begin{array}{l}\text { aktive und passive Mobilisation des } \\
\text { Handgelenks, Intensivierung der Narben- } \\
\text { behandlung, isometrische Muskelkräfti- } \\
\text { gung, ADL-Training und Erlernen von } \\
\text { Kompensationsmöglichkeiten in Bezug } \\
\text { auf die verringerte Beweglichkeit des } \\
\text { Handgelenks }\end{array}$ \\
\hline $8-12$ & $\begin{array}{l}\text { i. d. R. Entfernung der Kirschner-Drähte, } \\
\text { ergänzend zu den oben genannten } \\
\text { Behandlungsverfahren können nun auch } \\
\text { verstärkt manualtherapeutische Techni- } \\
\text { ken angewandt werden, Integration ins } \\
\text { Berufsleben }\end{array}$ \\
\hline 12 ff & \\
\hline
\end{tabular}

\section{Handtherapeutischer Befund}

Am 2. Juli 2019 begann Karl B. mit der ergotherapeutischen Behandlung. Zu diesem Zeitpunkt waren sieben Wochen seit der Operation vergangen. Postoperativ trat eine persistierende Schwellung von den Fingern bis in den Unterarm auf. Da sich diese durch Anlage des Castes verstärkte, entschied der Chirurg, den Cast zu spalten und mit einer physiotherapeutischen Lymphdrainage zu beginnen. Nach sechs Wochen Cast trug er eine Fertigorthese für weitere zwei Wochen, da sich im Röntgenbild eine nur unzureichende Knochenkonsolidierung zeigte. Das Handgelenk galt nach acht Wochen als bewegungsstabil und durfte aus der Schiene heraus beübt werden. In der ersten Einheit erstellte der Therapeut den in - Tab. 2 dargestellten Befund.

\section{„Ich möchte schnellstmöglich zurück in den Betrieb“}

Mit diesen Worten legte Karl B. zu Beginn der Therapie sein Rehaziel fest. Dafür war es wichtig, die Beweglichkeit des Handgelenks zu verbessern, das Narbengewebe für die Fingerbeweglichkeit zu mobilisieren und im Anschluss mit einem stabilisierenden Krafttraining zu 
- Tab.2 Eingangsbefund vom 02.07.2019

\begin{tabular}{|c|c|}
\hline Anamnese & $\begin{array}{l}59 \text { Jahre, Single, Schuhmacheraltge- } \\
\text { selle, seit Januar krankgeschrieben, } \\
\text { Händigkeit: rechts }\end{array}$ \\
\hline $\begin{array}{l}\text { relevante } \\
\text { Neben- } \\
\text { diagnosen }\end{array}$ & $\begin{array}{l}\text { Spaltung Karpaltunneldach 2017, } \\
\text { Depressionen, Diabetes Typ 2, Ulna- } \\
\text { Minus-Variante }\end{array}$ \\
\hline Inspektion & $\begin{array}{l}\text { deutliche Schwellung der gesamten lin- } \\
\text { ken Hand, Narbe flach und komplett } \\
\text { geschlossen, MCPs gerötet }\end{array}$ \\
\hline Palpation & $\begin{array}{l}\text { Druck ins Gewebe „teigiges Gefühl“, Nar- } \\
\text { bengewebe unelastisch und fest mit um- } \\
\text { und unterliegendem Gewebe } \\
\text { verwachsen }\end{array}$ \\
\hline Sensibilität & $\begin{array}{l}\text { Anästhesie um die Narbe auf einer Fläche } \\
\text { von } 2 \times 7 \mathrm{~cm} \text {, Hyposensibilität Kleinfinger } \\
\text { ulnarseitig }\end{array}$ \\
\hline Motorik & $\begin{array}{l}\text { Range of Motion (ROM) Handgelenk } \\
26^{\circ} / 10^{\circ} / 0^{\circ} \text { (Ex/ NN/Flex) } \\
0^{\circ} / 5^{\circ} / 5^{\circ} \text { (Ulnar-/NN/Radialduktion) } \\
\text { Fingerkuppen-Hohlhand-Abstand (FKHA) } \\
\text { Dig } 2-4,5 \mathrm{~cm} \\
\text { Dig } 3-4,0 \mathrm{~cm} \\
\text { Dig } 4-4,3 \mathrm{~cm} \\
\text { Dig } 5-2,5 \mathrm{~cm} \\
\text { Kraftdefizit }\end{array}$ \\
\hline $\begin{array}{l}\text { Schmerz- } \\
\text { befund }\end{array}$ & $\begin{array}{l}\text { kein Ruheschmerz, Belastungsschmerz in } \\
\text { den Fingern palmarseitig in Höhe der } \\
\text { Ringbänder A2 / A4, Schmerzen nach All- } \\
\text { tagsaktivitäten, abends mehr als mor- } \\
\text { gens, Ibuprofen } 600 \text { zwei bis drei Mal } \\
\text { täglich seit Januar } 2019\end{array}$ \\
\hline
\end{tabular}

beginnen. Ein gutes Ergebnis nach der erfolgten Operation ist i.d. R. ein ROM von 30/0/30. Das Problem meines Patienten war also vor allem die fehlende Flexion.

\section{Behandlungsmaßnahmen}

Ziel in den ersten zwei Wochen war es, die Durchblutung im Arm zu steigern, damit Muskel- und Bindegewebe sowie die Gelenke ausreichend mit Nährstoffen versorgt werden. Dazu haben sich Massagetechniken, wie z.B. eine Bindegewebemassage und aktive Bewegungsübungen, als effektiv erwiesen. Individuell angepasste Greifübungen von Materialien mit unterschiedlicher Größe und Struktur, wie z. B. Schaumstoffwürfel ( $>$ Abb.1) oder Murmeln, Sehnengleitübungen oder leichte Übungen mit Therapieknete zeigten schnell mobilisierende Wirkung.

Gleichzeitig begann ich mit einer moderaten Narbenbehandlung, die Karl B. zusätzlich im Heimprogramm durchführte. Um das Narbengewebe auf intensivere Techniken vorzubereiten, erfolgten Verschiebetechniken und Mobilisationsimpulse von der Narbe weg. Das Streichen bzw. Dehnen nach lateral ist für die Funktionalität

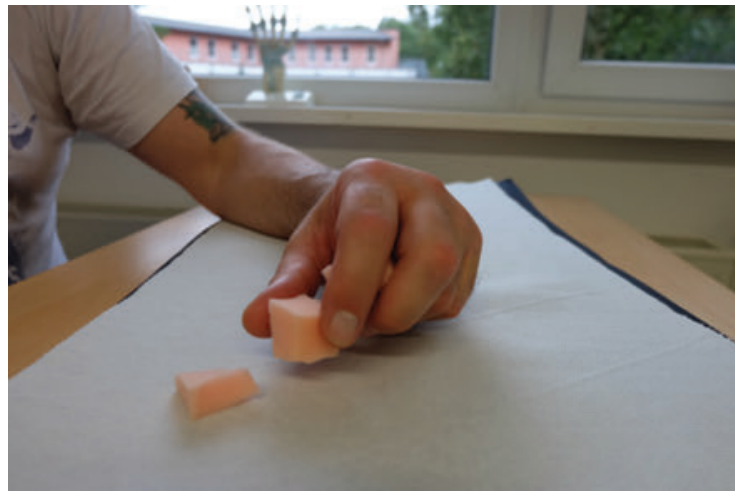

Abb. 1 Greifübungen mit Schaumstoffwürfeln. (Quelle: I. Vander)

effektiver, kann aber unter Umständen das Breitenwachstum anregen. Damit der Bewegungsreiz auch in die tieferen Hautschichten gelangt, sollte mit ausreichend Druck und grundsätzlich ohne Creme gearbeitet werden. Diese applizierte Karl B. unabhängig von der Narbenmobilisation drei bis fünf Mal täglich.

Ergänzend wendete ich die heiße Rolle, einen Low-LevelLaser und semizirkuläre Tapes auf Höhe der A2-Ringbänder an. Die heiße Rolle regt den Stoffwechsel an, fördert die Durchblutung, regt die Aktivität der Lymphbahnen an und wirkt schmerzlindernd. Aus diesen Gründen dient sie als sinnvoller Einstieg in die Behandlung. Der LowLevel-Laser wurde überwiegend im Bereich der schmerzhaften Gelenke (überwiegend PIPs) und im Verlauf der druckdolenten Beugesehnen angewandt ( Seine Wirkung basiert auf photochemischen Prozessen. Das Laserlicht soll eine leistungssteigernde Wirkung v.a. auf die Mitochondrien in der Zelle haben. Dadurch erhöhen sich Stoffwechselprozesse, eine antiphlogistische Wirkung setzt ein und eine Hemmung der Nozizeption findet statt. Es löst keinen thermischen Reiz aus und gilt daher als nebenwirkungsfrei. Die semizirkulären Tapes an den Fingern ( $\triangleright$ Abb.3) wirkten entlastend auf die schmerzhaften Beugesehnen und führten so zu einer mentalen Unterstützung bei der Greiftätigkeit im Alltag

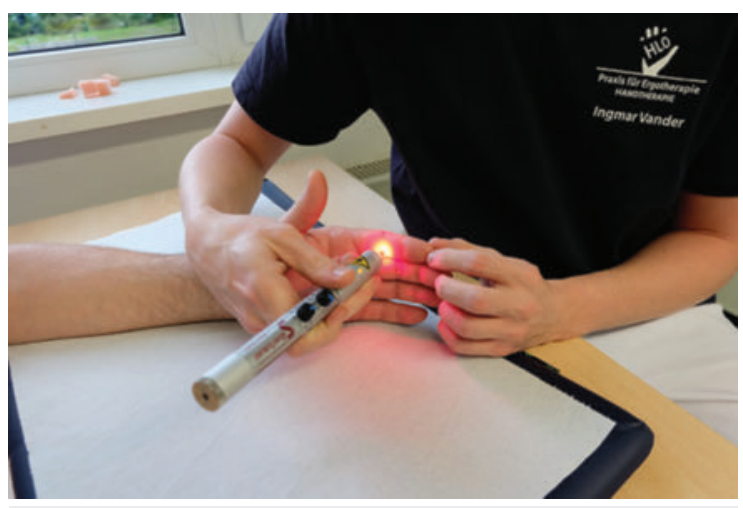

- Abb. 2 Low-Level-Laser. (Quelle: I. Vander) 


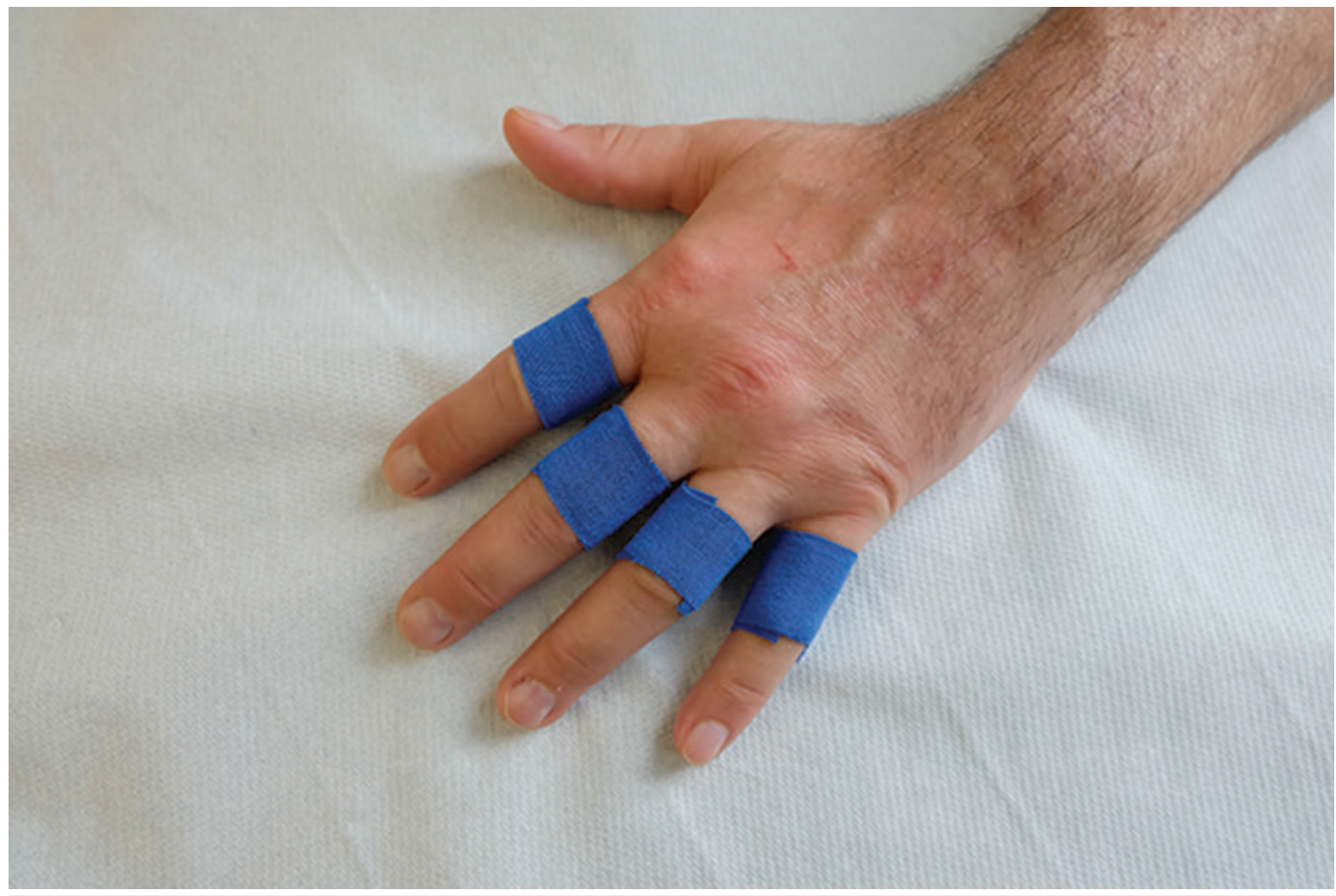

\Abb. 3 Semizirkuläre Tapes auf Höhe der A2-Ringbänder. (Quelle: I. Vander)

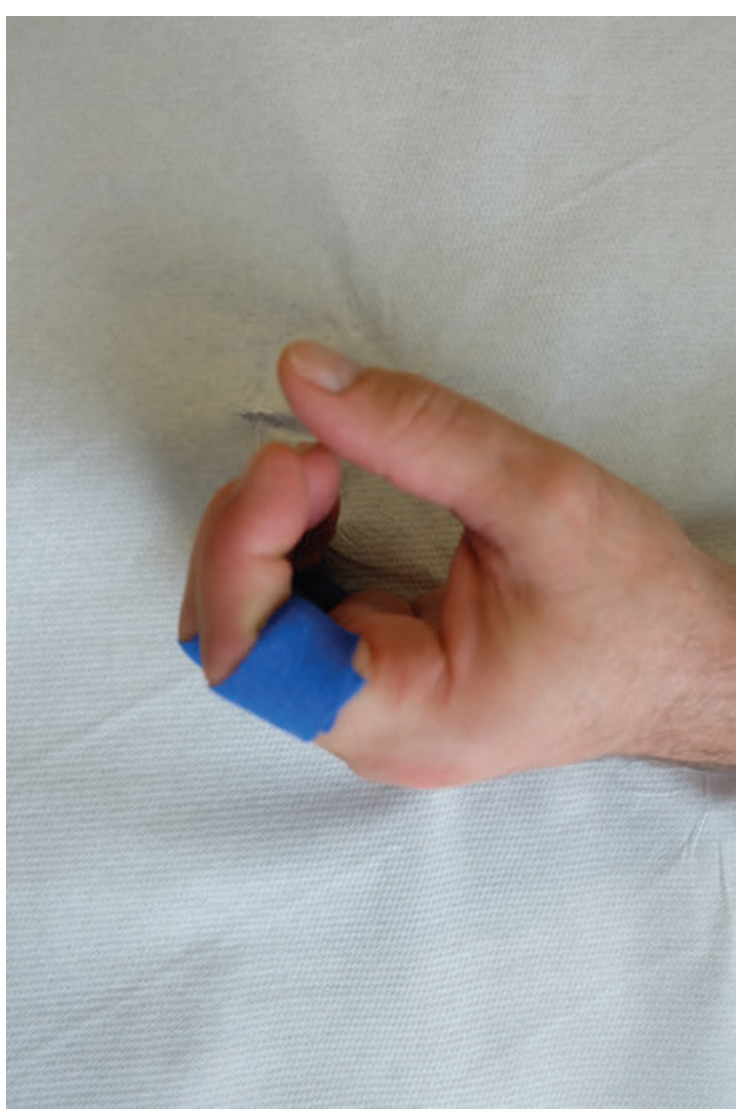

( Abb.4). Zu Hause führte Karl B. Handbäder im kalten Raps durch. Diese brachten ihm schnelle Linderung bei Schmerzen und Schwellung.

Am Ende der zehnten Woche konnten wir einen Zugewinn an Mobilität v.a. der Finger und einen deutlichen Rückgang der Schwellung feststellen ( Abb.5). Im DASH-Fragebogen erreichte Karl B. zu diesem Zeitpunkt einen Quick-DASH-Wert von 73,3 von 100 Punkten.

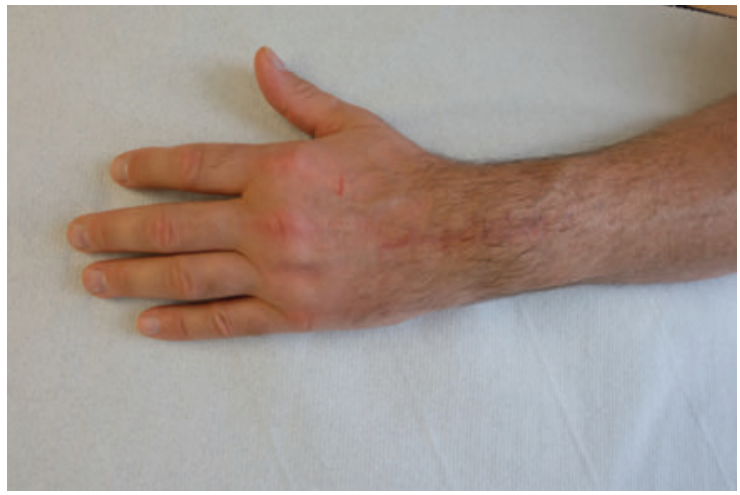

Abb. 5 Karl B.s Hand zehn Wochen postoperativ. (Quelle: I. Vander)

Abb. 4 Fingerflexion mit dem Tape. (Quelle: I. Vander) 


\section{ZUSATZINFO}

Durch Immobilisation des Handgelenks, Schmerzen und zusätzliche Schonhaltung wird der Stoffwechsel negativ beeinflusst. Die Folge ist eine herabgesetzte Durchblutung, durch die weniger Nährstoffe in die Extremität transportiert und weniger Stoffwechselabfallprodukte abtransportiert werden. Zusätzlich lagern bindegewebige Strukturen Wasser aus und Muskelmasse bildet sich zurück. Diese Prozesse führen zu den typischen, schmerzhaften Bewegungseinschränkungen nach längerer Ruhigstellung. Zu Beginn jeder Behandlung sollten deshalb stoffwechselfördernde Techniken angewandt werden, um das Gewebe auf die anschließende (dosierte) Mobilisation vorzubereiten.

\section{Zwei Schritte vor, einer zurück}

Ab der zehnten Woche führten wir nach Absprache mit dem Chirurgen zusätzlich Muskelkräftigungsübungen zur Stabilisierung des Handgelenks aus (ein Beispiel einer Stabilisationsübung mit dem Flexibar sehen Sie in - Abb. 6). Der Schwerpunkt lag dabei auf den M. flexor carpi ulnaris, dem M. extensor carpi ulnaris und den Mm. digitorum superficialis et profundus Strahl 4/5, um dem radialen Zug entgegenzuwirken.

Im Alltag fehlte es der Hand v.a. an Ausdauerkraft. Die Muskulatur ermüdete beispielsweise beim Aufräumen, Putzen oder Bogenschießen rasch und Karl B. nahm eine vermehrte Schwellug und Schmerzintensität wahr. Um die Ausdauerkraft zu steigern, wurde in der Therapie mit einer $0,5-\mathrm{kg}$-Hantel begonnen zu trainieren. Aufgabe war es, alle zwei Tage einen Dreiersatz mit jeweils 30-40 Wiederholungen der Handgelenksextension durchzuführen. Die Pausen zwischen den Tagen, an denen geübt wurde, dienten als Erholungsphasen für die Muskulatur.

Zu diesem Zeitpunkt kam Karl B. drei Mal in der Woche zur Behandlung. An Tagen, an denen er (vermutlich aufgrund seiner Depression) in schlechterer psychischer Verfassung war, berichtete Karl B. von vermehrt auftretenden Schmerzen und Schwellungen. Auch das Gefühl der Morgensteifigkeit in den Fingern sorgte für Verunsicherung. Da keine Arthrose der Finger bestand, ging ich davon aus, dass diese in der Festigkeit der Narbe und restlichen Schwellung begründet war. Ich konnte
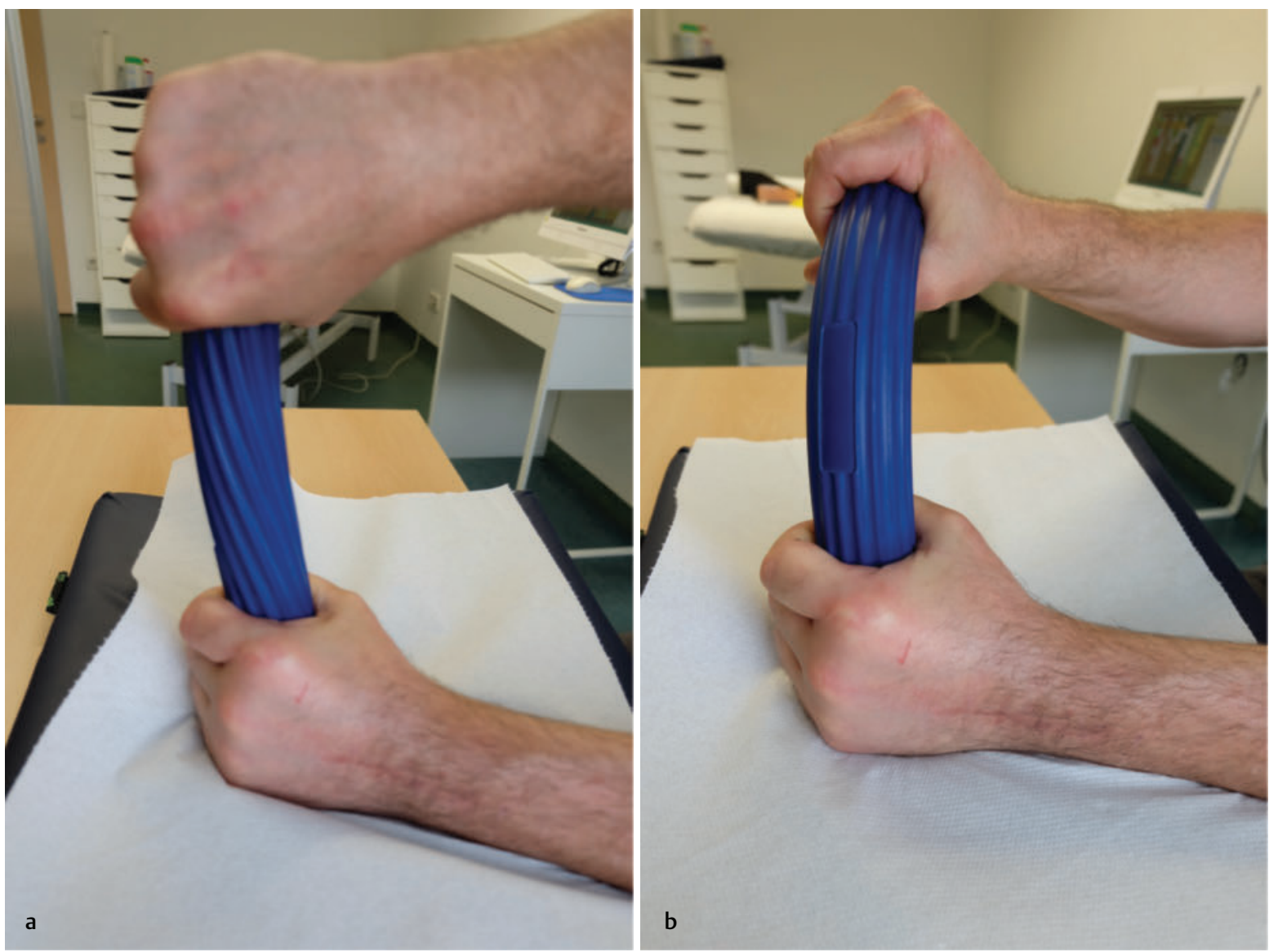

Abb. 6 Stabilisationsübungen mit dem Flexibar. (Quelle: I. Vander); a Extension/Flexion; b Pronation/Supination 
meinem Klienten die Angst etwas nehmen, indem ich ihm erklärte, dass viele Menschen nach einer Handoperation mit diesen Beschwerden zu tun haben und diese im Verlauf abnehmen sollten.

Auch auf zu viel Aktivität in der Therapie und im Alltag folgte eine Verschlechterung. Daher führten wir intensivere Trainingsformen wie Schwingstab und Übungen gegen höheren Widerstand nur an schmerzfreien Tagen durch. Dann rückten passive Verfahren, wie das Mobilisieren der Langfinger, leichte Traktion am Handgelenk und die Narbenmobilisation zurück in den Vordergrund. Im Gespräch stellte sich heraus, dass Karl B. privat unter vermehrtem Stress litt, der zu sehr wenig Schlaf in den Nächten führte. Der Zusammenhang zwischen psychischem und körperlichem Wohlbefinden bzw. einem regressiven Heilungsverlauf sollte in jeder Behandlung Beachtung finden. Der Therapeut muss in solchen Fällen gemeinsam mit dem Klienten und dem Arzt überlegen, ob eine alleinige Handtherapie überhaupt greifen kann oder eine zusätzliche psychologische Behandlung nötig ist. Auch der Austausch zwischen Therapeut und Arzt hinsichtlich des Übungsverhaltens und des damit verbundenen Fortschritts ist hierbei wichtig.

\section{Zwölf Wochen postoperativ}

Am 7. August, zwölf Wochen postoperativ, wurden alle Kirschner-Drähte vollständig entfernt. Im Röntgenbild stellte sich die Arthrodese vollständig konsolidiert dar ( Abb.7). Im Verlauf der Wundheilung kam es-begünstigt durch seinen Diabetes-zu einer oberflächlichen Infektion. Diese musste mit einem Antibiotikum behandelt werden. Zur Handtherapie kam Karl B. noch zwei bis drei Mal pro Woche, zusätzlich führte er die Lymphdrainage zwei Mal wöchentlich fort. Unter Berücksichtigung der sich verbessernden Wundverhältnisse wurden die Traktionsbehandlungen und die passive Mobilisation des Radiokarpalgelenks intensiver in die Palmarflexion durchgeführt. Das Handgelenk war deutlich besser zu flektieren, wenn die Finger nicht im Faustschluss gehalten wurden. Grund dafür waren die ausgeprägten Vernarbungen der synovialektomierten Strecksehnen. Daher war eine umfangreiche Narbenbehandlung nach erneutem Fadenzug unabdingbar. Zum Einsatz kamen der Dermoroller, die heiße Rolle, der Narbenstick und Schröpftechniken unter Gebrauch von Narbensalbe.

\section{Berufsspezifisches Übungsprogramm}

Als Handwerker verfügt Karl B. über eine gut ausgestattete Werkstatt in seinem Wohnhaus. Parallel zur ergotherapeutischen Behandlung begann er frühzeitig damit, verschiedene Arbeitsschritte aus seinem Berufsleben nachzustellen und zu üben. Beispielsweise führte er Schleifarbeiten durch oder nutzte den Hammer. Wöchentlich verbrachte er dadurch drei bis fünf Stunden zusätzlich mit einem sehr spezifischen Trainingsprogramm. Dabei fielen ihm die

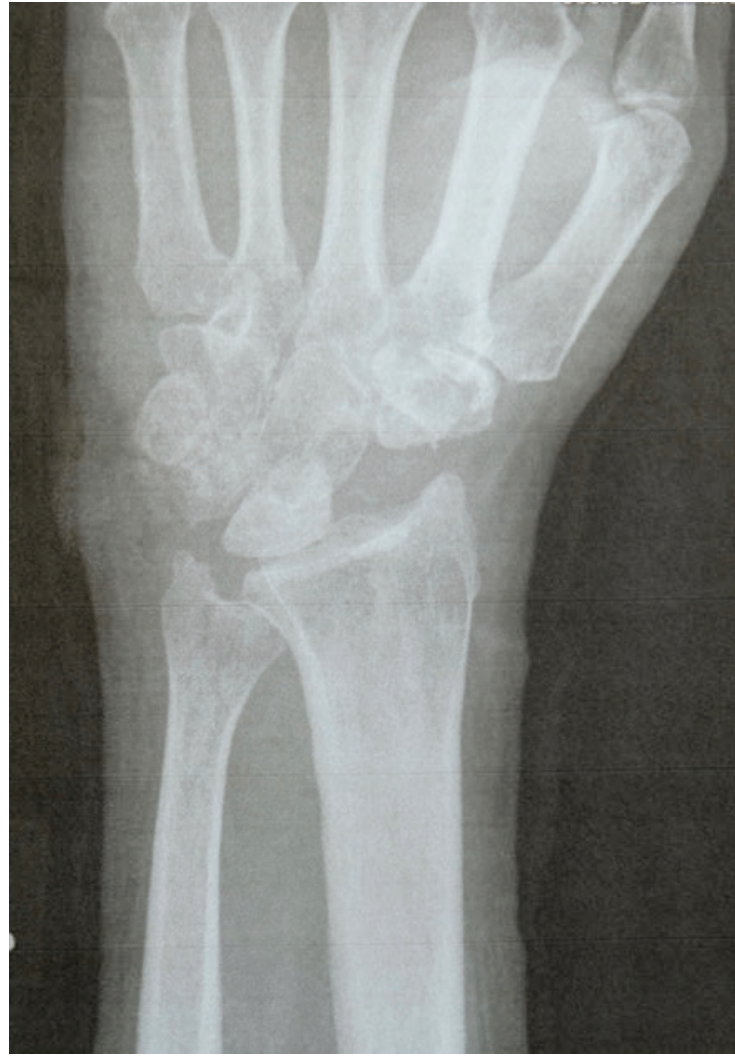

Abb. 7 Röntgenbild nach Materialentfernung am 07.08.2019. (Quelle: I. Vander)

fehlenden Funktionen der Hand auf. Im Rahmen der Therapie werteten wir sein Training aus dem Heimprogramm aus und konnten so das Anforderungsniveau anheben bzw. senken und neue Schwerpunkte setzen-beispielsweise Handgelenksflexion verbessern, Ausdauerkraft steigern und Radialdrift entgegenwirken.

\section{Zwischenbefund 14 Wochen postoperativ}

Dorsalextension und Palmarflexion verbesserten sich auf 30/0/5. Der Fingerkuppen-Hohlhand-Abstand verringert sich bei D2 um $0,5 \mathrm{~cm}$, bei D3 und D4 um $1,5 \mathrm{~cm}$ und bei D5 um $1 \mathrm{~cm}$. Die Kraftmessung mit dem Dynamometer ergab für links einen Wert von $12 \mathrm{~kg}$ und für rechts von $48 \mathrm{~kg}$. Die Sensibilitätsstörung dorsal/ulnar war nach der letzten Operation ausgeprägter. In der Ruheposition befand sich die Handgelenksstellung in einem leicht nach radial extendierten Winkel. Grund dafür könnte die zentrierende Wirkung der Beugemuskulatur auf das Handgelenk und das fehlende Skaphoid sein. Um dem entgegenzuwirken, leitete ich Karl B. an, gezielt nach palmar/ulnar zu üben. Die Narben erschienen oberflächlich unauffällig. Die Beweglichkeit im Gewebe zeigte sich im Vergleich zum Beginn verbessert, aber verhinderte weiterhin einen 
kompletten Faustschluss. Im Ruhezustand war die Hand wenig geschwollen. Bei bzw. nach Aktivität kam es aber zu einer spontanen Zunahme der Schwellung.

Im Alltag kam Karl B. immer besser zurecht. Er ging wieder zum Bogenschießen und berichtete von einer kontinuierlichen Leistungssteigerung der Hand. Im DASH-Fragebogen erreichte er nun einen niedrigeren Quick-DASH-Wert von 55.

In den nächsten Wochen sollte es darum gehen, die Mobilität und Kraft weiter zu steigern. Gezielt handwerkliche Tätigkeiten bzw. Adaptionen durch therapeutische Medien sollten die Therapie möglichst individuell und lebensnah gestalten. Nach der 16. Woche beendete Karl B. jedoch die ambulante Ergotherapie in unserer Einrichtung aufgrund einer Anordnung der Berufsgenossenschaft. Er begann mit einer Rehamaßnahme in einer Unfallbehandlungsstelle. Dort absolviert er seit einigen Wochen ein Therapieangebot über vier bis sechs Stunden täglich. Ziel ist es, dass Karl B. gegen Ende 2019 wieder voll in seinem Beruf einsteigen kann.

\section{Abschlussbefund nach 22 Wochen}

Nach 22 Wochen bekam ich die Möglichkeit, ihn noch mal zu sehen und mir ein Bild vom Fortschritt seiner Handfunktion zu machen:

Das Range of Motion hat sich in allen Freiheitsgraden verbessert. Die Extension gelingt bis $40^{\circ}$ und liegt damit $10^{\circ}$ über der Erwartung. Die problematische Flexion konnte auf $10^{\circ}$ gesteigert werden. Ulnar- und Radialduktion verbesserten sich ebenfalls auf 5/0/10. Der Fingerkuppen-Hohlhand-Abstand beträgt am Zeigefinger noch $1,5 \mathrm{~cm}$, die anderen Fingerkuppen erreichen die Hohlhand. Die Handkraftmessung hat sich in der betroffenen Hand nochmals um $26 \mathrm{~kg}$ gesteigert und liegt damit nur noch ca. $10 \mathrm{~kg}$ unter der gesunden Hand. Die Sensibilität stellt sich unverändert dar. Die große dorsale Narbe über dem Handgelenk ist weich, beweglich und schmerzfrei. Die ulnaren Narben (Materialentfernung) sind noch verhärtet und druckdolent. Der DASH-Wert war nochmals um ca. 14 Punkte gesunken. Im Alltag fühlt sich Karl B. kaum noch eingeschränkt. Er fährt wieder viel Fahrrad und steigert seine Leistungsfähigkeit beim Bogenschießen zufriedenstellend. Nach der Rehamaßnahme wird Karl B. mit dem Hamburger Modell wieder in seinen Beruf einsteigen. $\mathrm{Er}$ ist optimistisch, dass er seine Arbeitsleistung stetig steigern wird, und freut sich darauf, in den Beruf zurückzukehren. Die letzte Therapieeinheit beendete er mit den Worten: „Die Operation hat sich jetzt schon gelohnt.“

\section{Autorinnen / Autoren}

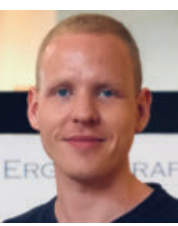

\section{Ingmar Vander}

Ergotherapeut und zertifizierter Handtherapeut, seit 2012 hauptsächlich im Bereich Handtherapie tätig, seit 2014 fachliche Leitung der HLO Handtherapie im Krankenhaus Waldfriede in Berlin-Zehlendorf.

\section{Korrespondenzadresse}

\section{Ingmar Vander}

HLO Handtherapie H. Lorenzen \& Mitarbeiter

Argentinische Allee 40

14163 Berlin

E-Mail: ingmar.v@me.com

\section{Literatur}

[1] Eigene Nachbehandlungsrichtlinien aus unserer Praxis. In individueller Absprache mit den Ärzten

[2] Krimmer H., Lanz U. Mediokarpale Teilarthrodese des Handgelenks. Operat Orthop Traumatol 1996; 8: 175-184

CALL FOR PAPERS

Auch Sie können in der Praxis Handreha publizieren.

Wir freuen uns über Fallbeispiele und Fachartikel rund um das Thema Handtherapie.

Senden Sie Ihren Artikel an praxis.handreha@thieme.de

Über die Annahme der Artikel entscheidet das Herausgeberteam.

Publizierte Artikel werden honoriert. 